\title{
A Multifunctional Microfluidic Device for Blood Typing and Primary Screening of Blood Disease
}

\author{
Jia-Hui Lin, ${ }^{\dagger}{ }^{\prime \prime}$ Tsung-Ting Tsai, ${ }^{\ddagger}$ Qiang Zeng, ${ }^{\dagger}$ Chun-Yen Chang, ${ }^{\dagger}$ \\ Jun-Yu Guo, ${ }^{\dagger}$ Chi-Jui Lin, ${ }^{\S}$ and Chien-Fu Chen ${ }^{\dagger^{*}}$ \\ †Institute of Applied Mechanics, National Taiwan University, Taipei 106, Taiwan \\ *Department of Orthopaedic Surgery, Bone and Joint Research Center, Chang Gung Memorial \\ Hospital and Chang Gung University College of Medicine, Taoyuan 333, Taiwan \\ ${ }^{\S}$ Department of Laboratory Medicine, Chang Gung Memorial Hospital, Taoyuan 333, Taiwan
}

\author{
*Corresponding author \\ Prof. Chien-Fu Chen \\ E-mail: stevechen@ntu.edu.tw
}


$A_{\text {(a) }}$

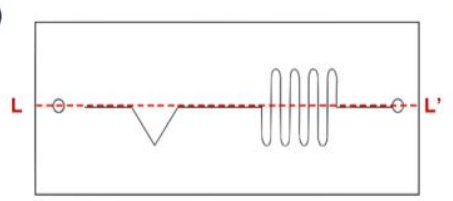

(b)

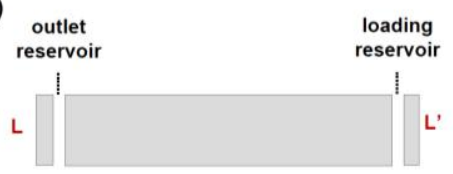

(c)

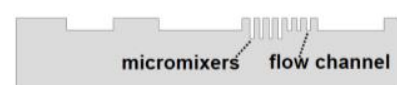

(d)

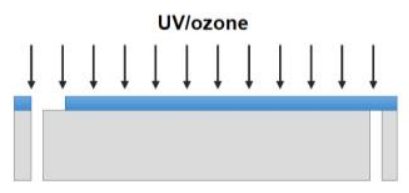

(e)

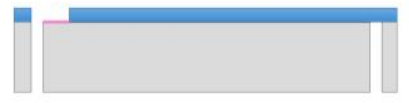

(f)

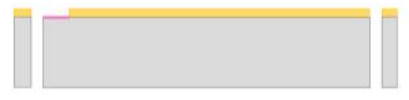

(g)

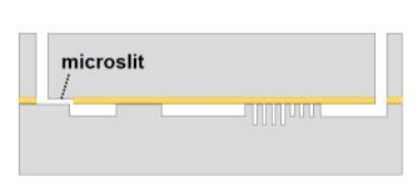

(a)

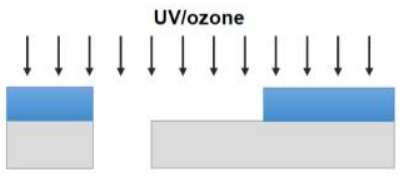

(b)

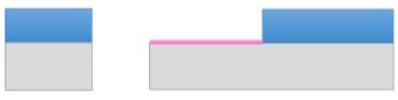

(c)

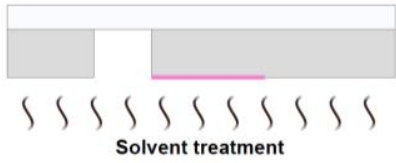

(d)

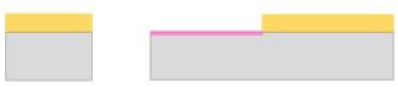

Figure S1. A schematic illustration (not to scale) of the fabrication process of the single-channel microfluidic chip using the selective solvent swelling method. (A) The fabrication process of the chip. (a) Top view of the single-channel chip. (b) The top cover chip is drilled to form two holes for a loading reservoir and an outlet reservoir. (c) The bottom chip is milled to form the serpentine microchannels, micromixers, and a check-shaped channel. (d)(e) The top cover chip is masked with tape and exposed to UV/ozone to form the surface-modified area. (f) After removing the mask, the top cover chip is swelled with cyclohexane vapor. (g) The top and bottom chips are sealed into a single-channel chip by pressing them at room temperature. (B) The selective solvent swelling method is used to form a microslit. (a)(b) After exposing the masked region of the polymeric chip surface to UV/ozone, the unmasked region is modified with hydrophilic groups. (c)(d) The chip surface is treated with cyclohexane vapor, causing the unmodified region of COP chip to absorb the non-polar solvent and swell, while the UV/ozone modified surface resists the absorption of solvent vapor. 

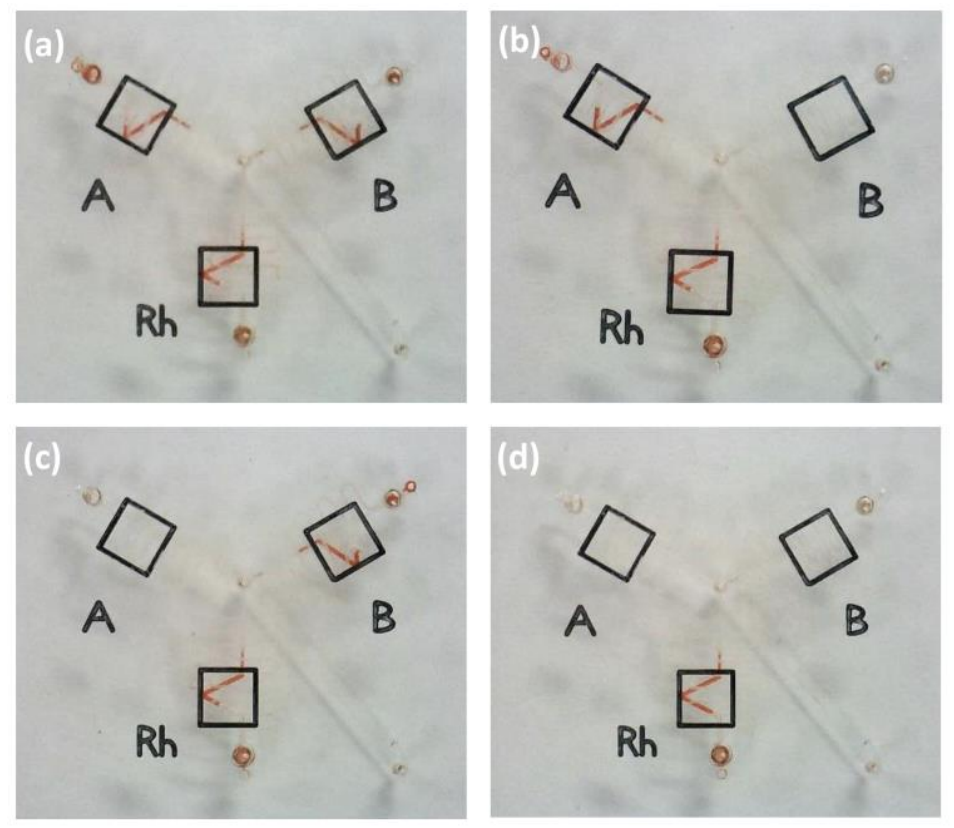

Figure S2. Representative chip test results are shown for (a) AB Rh+, (b) A Rh+, (c) B Rh+, and (d) $\mathrm{O} R \mathrm{R}+$ blood types. These results are clearly visualized by the red lines corresponding to the A, B, and Rh symbols on the top of the chip.

Table S1. The length of agglutinated red blood cells formed in multifunctional microfluidic device for clinical blood samples with different hematocrit levels

\begin{tabular}{cccc}
\hline Blood sample & Sample size & Length of red line $(\mathrm{cm})$ & Hematocrit $(\%)$ \\
\hline Normal ABO groups & 8 & $0.89 \pm 0.04$ & $41.5 \pm 2.5$ \\
PV & 8 & $1.24 \pm 0.21$ & $55.8 \pm 5.8$ \\
Anemia & 8 & $0.38 \pm 0.10$ & $25.5 \pm 2.4$ \\
ABO subgroups & 6 & $0.03 \pm 0.02$ & $30.3 \pm 6.6$ \\
\hline
\end{tabular}



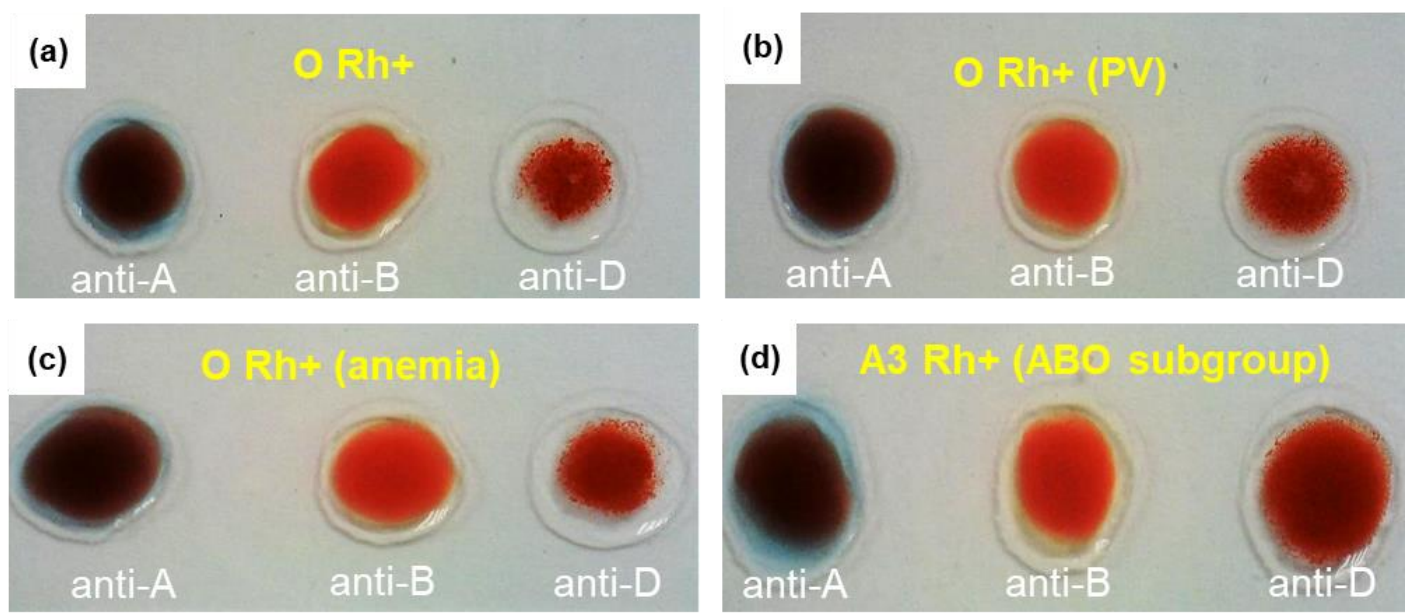

Figure S3. Representative agglutination results of (a) $\mathrm{O} \mathrm{Rh}+$ (normal groups), (b) $\mathrm{O} \mathrm{Rh}+(\mathrm{PV})$, (c) $\mathrm{O} \mathrm{Rh}+$ (anemia), and (d) $\mathrm{A} 3 \mathrm{Rh}+(\mathrm{ABO}$ subgroup), as determined by the slide test.

Video S1. The process of blood typing and disease screening using a multifunctional microfluidic chip. A drop of blood obtained from a finger prick is loaded into the reservoir, followed by pulling the rod of the syringe to generate negative pressure, which drives the blood flow into the microchannels, where red blood cells can interact with the preloaded antibodies (anti-A, anti-B, and anti-D) in the micromixers. 